\title{
Analyse und Quantifizierung der Umweltaspekte von Fördermitteln in der Intralogistik
}

\section{Analysis and Quantification of Environmental Aspects in Intra-Logistics Systems}

\author{
Timo Schilling *, Alexander Frenkel *, Rainer Bruns * \\ Matthias Amberger **, Gabriel Fischer ${ }^{\star \star}$, Willibald A. Günthner ${ }^{* \star}$ \\ Meike Braun ${ }^{\star \star \star}$, Frank Schönung ${ }^{\star \star \star}$, Kai Furmans ${ }^{\star \star \star}$ \\ * Lehrstuhl für Maschinenelemente und Technische Logistik (MTL) \\ Helmut-Schmidt-Universität / Universität der Bundeswehr Hamburg \\ ** Lehrstuhl für Fördertechnik Materialfluss Logistik (fml) \\ Technische Universität München \\ *** Institut für Fördertechnik und Logistiksysteme (IFL) \\ Karlsruher Institut für Technologie
}

D as Projekt „Analyse und Quantifizierung der Umweltauswirkungen von Fördermitteln in der Intralogistik“ hat sich zum Ziel gesetzt, die Umweltaspekte verschiedener Fördermittel während des gesamten Lebenszyklus zu analysieren, zu quantifizieren und zu bewerten. Dazu werden für die Produktgruppen Flurförderzeuge, Krane \& Hebezeuge und Lagertechnik Methoden entwickelt, um die Umweltaspekte, die von diesen Geräten bzw. Systemen zu erwarten sind, einer genaueren Untersuchung zu unterziehen.

[Schlüsselwörter: Umweltaspekte, Grüne Logistik, Flurförderzeuge, Krane, Lagertechnik]

$\mathbf{T}$ he research project "Analysis and quantification of environmental impacts of conveyors in the intralogistics sector" aims for analysis, quantification and evaluation of environmental aspects during the whole lifecycle of different conveyors. Therefore methods to examine the environmental aspect of industrial trucks, cranes and lifting equipment and storage equipment are being developed.

[Keywords: environmental aspects, green logistics, industrial trucks, cranes, storage equipment]

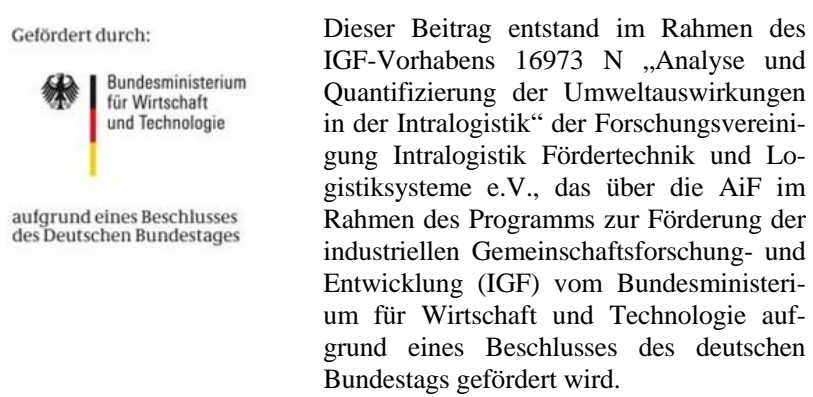

\section{EINLEITUNG}

Die anhaltenden Diskussionen in der Öffentlichkeit über Ressourcenverfügbarkeit, Klimawandel und Energieeinsparung haben bereits viele Bereiche des alltäglichen Lebens erfasst. Schlagworte wie Energieeffizienz, Carbon Footprint oder Life Cycle Analysis sind allgegenwärtig, wobei für einige Produkte bereits Vorgaben hinsichtlich ihrer Energieeffizienz gemacht wurden. [Gün09] haben sich bereits diesem Trend in der Logistik gewidmet. Erste Ansätze zur Untersuchung der Umweltaspekte bei Fördermitteln in der Intralogistik können einer Ökobilanz der Firma Jungheinrich [Jun12] entnommen werden.

Eine ganzheitliche Untersuchung der Fördermittel in der Intralogistik hinsichtlich ihrer Umweltleistungsfähigkeit existiert bisher nicht. Deshalb wird dazu in einem ersten Schritt eine umfassende Liste von Umweltaspekten erstellt, die von den untersuchten Produktgruppen verursacht werden. Nach [DIN09] ist ein Umweltaspekt als „Bestandteil, der Tätigkeiten, Produkte oder Dienstleistungen einer Organisation, der auf die Umwelt einwirken kann“ definiert. Eine Umweltauswirkung dagegen ist ,jede Veränderung der Umwelt, ob ungünstig oder günstig, die sich ganz oder teilweise aus Umweltaspekten einer Organisation ergibt“ [DIN09]. Die Wechselwirkungen zwischen Umweltaspekten und Umweltauswirkungen werden in Abbildung 1 gezeigt.

Die Höhe der Umweltaspekte wird durch vielfältige Faktoren beeinflusst. Beispielsweise ergibt eine Veränderung der Einschaltzeit einer Anlage auch eine Änderung des Energiebedarfs oder bei Verbrennungsmotoren eine Änderung des Ausstoßes von Kohlenstoffdioxid. Die Höhe der Umweltaspekte beeinflusst wiederum die Umweltauswirkungen. So wird einer Erhöhung des Ausstoßes von Kohlenstoffdioxid eine dementsprechende Veränderung des Klimas als Umweltauswirkung zugerechnet. 


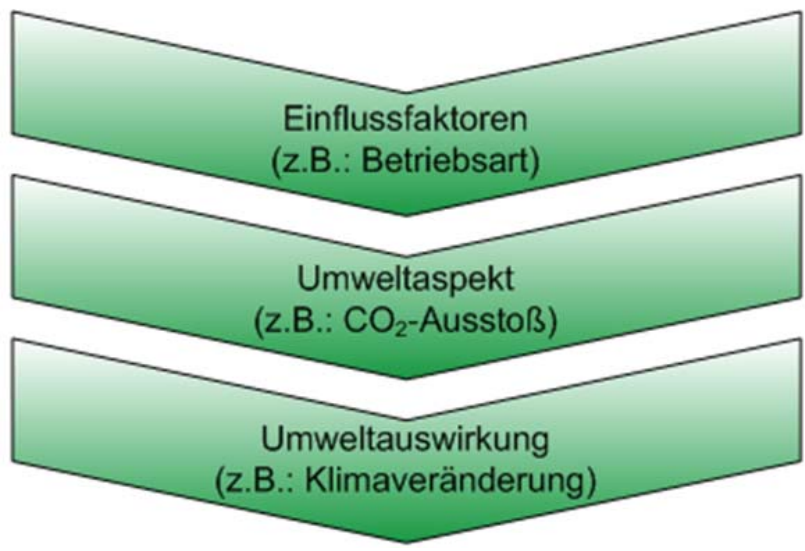

Abbildung 1. Wechselwirkungen zwischen Umweltaspekten und Umweltauswirkungen

Den Untersuchungen innerhalb des Forschungsprojekts liegt der vollständige Lebenszyklus der Produkte nach [EU05] mit den in Abbildung 2 dargestellten Produktlebensphasen zugrunde. Für jede dieser Phasen wird die Höhe der einzelnen Umweltaspekte abgeschätzt und bewertet.

\begin{tabular}{|l|l|}
\hline 1 & - Auswahl und Einsatz von Rohmaterial \\
\hline 3 & - Fertigung \\
\hline 4 & - Installierung und Wartung \\
\hline 5 & - Nutzung \\
\hline 6 & - Ende der Lebensdauer \\
\hline
\end{tabular}

\section{Abbildung 2. Lebenszyklus nach [EU05]}

Die Einteilung der Umweltaspekte nach der Art der Einwirkung ist in Tabelle 1 mit einigen Beispielen dargestellt. Grundsätzlich wird dabei zwischen Einwirkungen auf Luft, Boden und Wasser unterschieden, sowie zwischen Bedarf an bzw. Einsatz von Ressourcen und der Entstehung von Abfällen. Dabei gilt es zunächst abzuschätzen, welche Lebensphase für den größten Anteil der Umweltaspekte der Fördermittel verantwortlich ist, um in einem nächsten Schritt denkbare Verbesserungsansätze zur Reduzierung der Höhe der Umweltaspekte abzuleiten und zu prüfen.
Tabelle 1. Kategorien der Umweltaspekte

\begin{tabular}{|c|c|}
\hline $\begin{array}{l}\text { Emissionen } \\
\text { in die Luft }\end{array}$ & $\begin{array}{l}\text { - Treibhausgase } \\
\text { - Schadstoffe } \\
\text { - Staub } \\
\text { - . . . }\end{array}$ \\
\hline $\begin{array}{l}\text { Emissionen } \\
\text { in das Wasser }\end{array}$ & $\begin{array}{l}\text { - Nährstoffanreicherung } \\
\text { - Schadstoffe } \\
\text { - ... }\end{array}$ \\
\hline $\begin{array}{l}\text { Wirkungen auf } \\
\text { den Boden und die } \\
\text { Fläche }\end{array}$ & $\begin{array}{l}\text { - Flächennutzung und } \\
\text { Bodenbeanspruchung } \\
\text { - Erschütterungen und } \\
\text { Schwingungen } \\
\text { - . . }\end{array}$ \\
\hline $\begin{array}{l}\text { Einsatz / Verbrauch } \\
\text { von Ressourcen }\end{array}$ & $\begin{array}{l}\text { - Rohstoffe } \\
\text { - Hilfsstoffe } \\
\text { - Betriebsstoffe } \\
\text { - . . }\end{array}$ \\
\hline $\begin{array}{l}\text { Entstehung } \\
\text { von Abfall }\end{array}$ & $\begin{array}{l}\text { - Verwertbar } \\
\text { - Wiederverwendbar } \\
\text { - ... }\end{array}$ \\
\hline
\end{tabular}

In den folgenden Abschnitten werden die jeweiligen Schwerpunkte aus der Arbeit der am Projekt beteiligten Forschungsinstitute vorgestellt.

\section{UMWELTASPEKTE VON FLURFÖRDERZEUGEN (MTL)}

Der Trend zu einer steigenden Sensibilität für umweltfreundliche Produkte betrifft heute fast alle Produktgruppen. Während für einige Produkte bereits fundierte Informationen über ihre Umweltauswirkungen vorliegen, sind andere Produktgruppen noch nicht umfassend betrachtet worden. Das Ziel des hier vorgestellten Teilprojektes ist vor allem eine Aussage zu den Umweltaspekten, wie z. B. Energiebedarf bzw. Treibhausgas-Ausstoß, aller Flurförderzeuge in der Europäischen Union. Flurförderzeuge sind bodengebundene Unstetigförderer und existieren in einer Vielzahl verschiedener Ausführungen. Die DIN ISO 5053 [DIN94] unterteilt Flurförderzeuge nach Benutzungsart, Antriebsarten, Art der Steuerung, Hubhöhe und Fahrbewegungen. Sie nennt u.a. Schlepper, Hubwagen und Gabelstapler. Das Einsatzspektrum von Flurförderzeugen ist selbst unter vergleichbaren Typen sehr unterschiedlich.

Entsprechend der methodischen Herangehensweise ist zuerst der Produktlebenszyklus von Flurförderzeugen beschrieben worden. Anschließend ist anhand ausgewählter Beispiele abgeschätzt worden, welche Relevanz die Umweltaspekte in den einzelnen Lebensphasen haben, um die vorrangig zu untersuchende Lebensphase zu identifizieren. Aus den Absatzzahlen werden die europäischen Bestände an Flurförderzeugen berechnet. Auf dieser Basis werden unter Verwendung eines standardisierten Fahrzyklus` mit Hilfe definierter Faktoren die Umweltaspekte der europäischen Flurförderzeugflotte berechnet. 


\subsection{Die ProdukTLEBENSPHASEN VON FLURFÖRDERZEUGEN}

Grundsätzlich soll das gesamte Produktleben von Flurförderzeugen in die Betrachtung einbezogen werden. Dieses besteht - vergleichbar mit anderen Produkten - aus Rohstoffgewinnung, Produktion, Transport, Betrieb/ Nutzung und Entsorgung (in Anlehnung an [EU05], [DIN06]). Projektintern ist der Lebenszyklus von Flurförderzeugen leicht abgewandelt zusammengefasst worden (s. Abbildung 3). Aufgrund mangelnder Daten und Konzepte im Bereich der Entsorgung von Flurförderzeugen ist diese Lebensphase vorerst aus der Betrachtung ausgeschlossen worden.

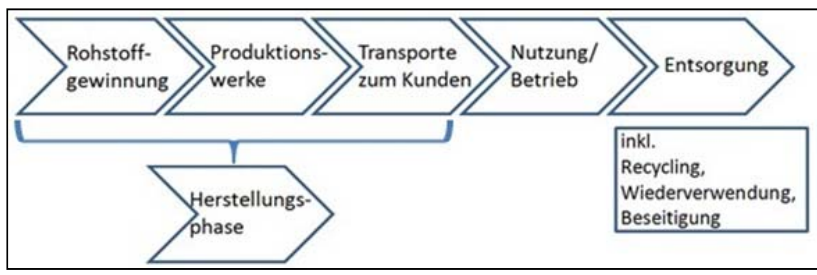

Abbildung 3. Lebensphasen von Flurförderzeugen, in Anlehnung an [EU05], [DINO6]

\subsection{BenÖtigte ANNAHMEN}

Um an die o. g. Zielstellung heranzugehen, sind einige Annahmen notwendig, die teils auf recherchierten Daten und teils auf Erfahrungswerten der am Projekt beteiligten Unternehmen beruhen. Als Erfahrungswert der Flurförderzeughersteller wird unterstellt, dass ein Flurförderzeug-Leben durchschnittlich 10.000 Betriebsstunden beträgt. Diese verteilen sich über acht Jahre (aufgrund der Abschreibungsmöglichkeit nach Afa-Tabellen [Bun12]).

Um Aussagen über die Umweltaspekte der Produktion treffen zu können, sind mehrere Angaben notwendig. Neben der Anzahl der pro Jahr in den Markt gebrachten Fahrzeuge ist die Masse jedes einzelnen Fahrzeugs zur Bestimmung der Auswirkungen der verwendeten Werkstoffe notwendig. Allerdings lässt sich - wie oben bereits beschrieben - die genaue Anzahl einzelner Modelle nicht bestimmen. Zudem sind die Produktionsverfahren einzubeziehen. Da diese Angaben schwer zu verallgemeinern sind, werden vorerst die im Rahmen des Projekts erhobenen Daten (technische Daten, Verbrauchsangaben usw.) als allgemeingültig angenommen.

\subsection{BESTANDSERMITTLUNG}

Grundsätzlich wichtig zur Hochrechnung der Umweltaspekte aller Lebensphasen sind die jährlichen Absatzzahlen, der Gesamtbestand und die jährlich aus dem Markt austretenden Fahrzeuge. Die jährlichen Absatzzahlen - aufgeschlüsselt nach Flurförderzeugtyp und Tragfähigkeitsklasse - sind bekannt. Um den Gesamtbestand zu erhalten, werden die Absatzzahlen gemittelt und mit der durchschnittlichen Lebensdauer multipliziert. Die Anteile der einzelnen Hersteller und die der jeweiligen Modelle können allerdings aufgrund der Datenmenge und der Tatsache, dass viele Hersteller diese Informationen nicht preisgeben, nicht ermittelt werden.

\subsection{VDI-ARBEITSSPIEL}

Weiterhin muss der Energiebedarf der einzelnen Fahrzeuge bekannt und aufgrund der Vergleichbarkeit auf einheitliche Weise gemessen sein. Aus dem Energieberdarf lassen sich einige wesentliche Umweltaspekte berechnen. In den Typenblättern von Flurförderzeugen nach VDI-Richtlinie 2198 [VDI02] ist eine Verbrauchsangabe nach einem standardisierten Arbeitsspiel zu finden (siehe Abbildung 4). Dabei fährt das Flurförderzeug mit Nennlast zwischen den Punkten A und B (Abstand je nach Flurförderzeugtyp - bei Gabelstaplern $30 \mathrm{~m}$ ) und hebt diese jeweils bei A und $\mathrm{B}$ an.

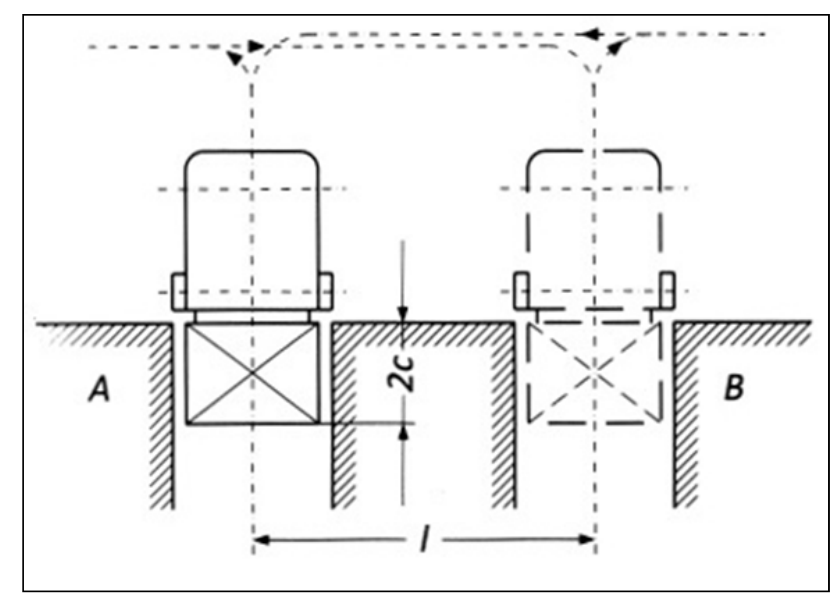

Abbildung 4. Arbeitsspiel nach VDI-Richtlinie 2198 [VDI02]

Diese Verbrauchswerte sind für viele Flurförderzeuge öffentlich zugänglich und sind als Grundlage für die Verbrauchsberechnung herangezogen worden. Darauf aufbauend werden einerseits energieträgerspezifische $\mathrm{CO}_{2}$ Faktoren recherchiert, andererseits wird geprüft, inwiefern Ladefaktoren für elektrische Energiespeicher einbezogen werden müssen. Da für viele Hersteller bzw. Modelle keine VDI-Verbrauchswerte angegeben werden und die ermittelten Werte teilweise erheblich schwanken, werden bei der Auswertung alle ermittelten minimalen und maximalen Werte ausgegeben.

Abbildung 5 zeigt beispielhaft die recherchierten Verbrauchsangaben nach VDI-2198 für Dreirad-ElektroGegengewichtstapler. Auch für die weiteren Typen sind die aufgenommenen Verbräuche teils sehr unterschiedlich. Es kann nicht zweifelsfrei angegeben werden, wie häufig die einzelnen Verbräuche am Markt auftreten. Zur transparenten Berücksichtigung dieser Problematik werden die Umweltaspekte, die sich aus den Energiebedarfen bzw. Kraftstoffverbräuchen ergeben, in einer Spanne zwischen minimalen und maximalen Verbäuchen angegeben. 


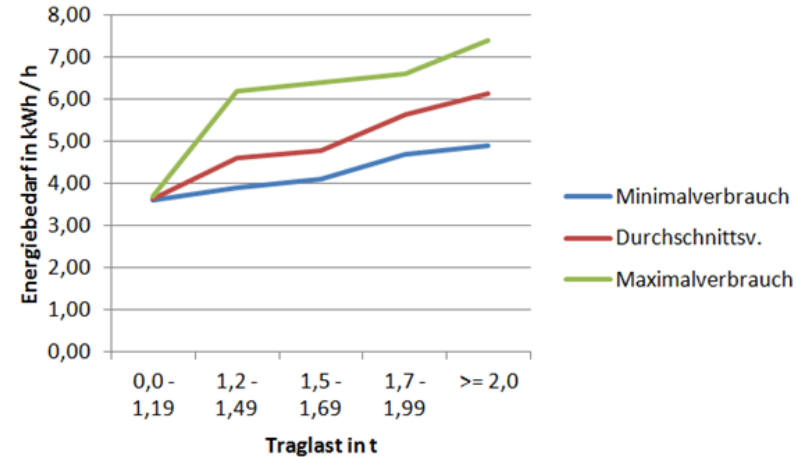

Abbildung 5. VDI-Verbräuche am Beispiel von 3-RadElektro-Gegengewichtstaplern

\subsection{BEWERTUNG DER RELEVANZ DER EINZELNEN LEBENSPHASEN}

Vor der detaillierten Betrachtung ist abgeschätzt worden, wie hoch der Energiebedarf in den Lebensphasen Herstellung und Nutzung ist. In der Phase ,Produktionswerke' werden Fertigung und Montage der Hersteller erfasst. Zur Ermittlung des Energiebedarfs werden Daten von drei exemplarischen Produktionswerken verwendet (vor allem Energiebedarfsdaten und Ausbringungsmengen). Die Systemgrenzen der Produktion von Flurförderzeugen schließen die Gewinnung und Bearbeitung der Rohstoffe sowie die Tätigkeiten des Herstellers (OEM) ein. In der Supply Chain vorgelagerte Schritte sind nicht Teil der Betrachtung.

In Fertigung und Montage der Hersteller sind allerdings Energiebedarfe der Rohstoffgewinnung und verarbeitung nicht enthalten. Zu deren Ermittlung sind die prozentual höchsten Werkstoffanteile je Flurförderzeugtyp abgeschätzt und entsprechende $\mathrm{CO}_{2}$-Faktoren recherchiert worden (z. B. nach [Öko12]).

In einer ersten fundierten Abschätzung anhand konkreter Beispiele lassen sich vorläufige Ergebnisse erkennen. Es zeigt sich, dass die Nutzungsphase mit 81 bis $88 \%$ den höchsten Anteil am Energiebedarf hat (siehe Abbildung 6). Obwohl nicht alle Einflüsse umfassend berücksichtigt werden können und die getroffenen Annahmen mit erheblichen Unsicherheiten behaftet sind, liefert das hier beschriebene Modell bereits einen guten Eindruck der prozentualen Anteile der Lebensphasen am Energiebedarf.

Wenn in dieses Modell beispielsweise die Produktionswerke der Zulieferer und die Transporte für Anlieferungen von Zukaufteilen mit einbezogen würden, könnte u. U. der Anteil der Herstellungsphase ansteigen. Aus diesem Grund ist die Herstellungsphase tendenziell unterbewertet. Aufgrund der vorherigen Ergebnisse ist aber davon auszugehen, dass der Anteil der Herstellungsphase am gesamten $\mathrm{CO}_{2}$-Ausstoß $20 \%$ nicht übersteigt. Dieses Teilprojekt konzentriert sich folglich auf die Auswertung des Betriebs bzw. der Nutzung von Flurförderzeugen.

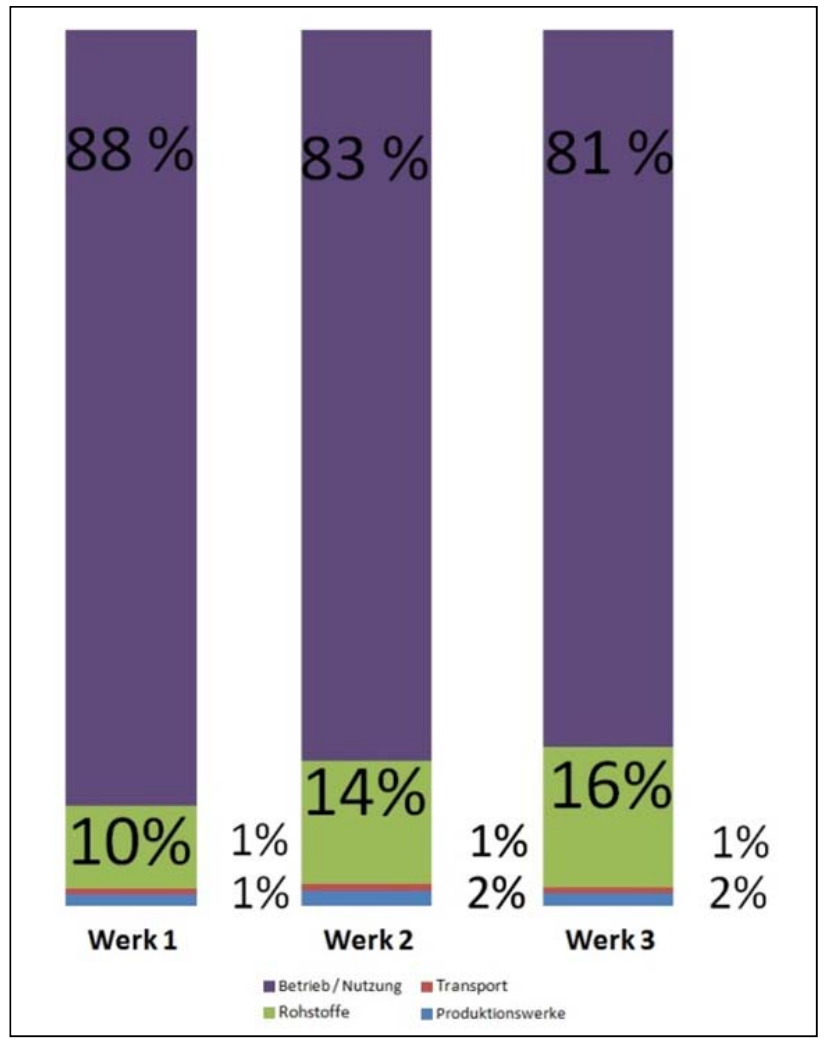

Abbildung 6. Anteile der Lebensphasen am CO2-Ausstoß

\subsection{EIN BLICK AUF DIE ERGEBNISSE / ABLEITUNG WEITERER UMWELTASPEKTE}

Zur Ermittlung des Gesamtbestandes an Flurförderzeugen in der Europäischen Union sind jährliche Absatzzahlen ausgewertet worden. Der ermittelte Durchschnitt über mehrere Jahre wird mit der angenommenen Lebensdauer multipliziert. Dabei sind alle Flurförderzeugtypen, die einen Marktanteil von weniger als 5 \% haben, im späteren Verlauf der Studie nicht weiter betrachtet worden. Durch diese Vorgehensweise ist es gelungen, über $80 \%$ des Gesamtmarktes zu erfassen und gleichzeitig Geräte, die in der VDI-Richtlinie nicht enthalten sind (z. B. Schmalgangstapler), auszublenden.

Unter Verwendung der Daten aus der Recherche der Verbrauchswerte für Dreirad-Elektro-Gegengewichtsstapler (siehe Abbildung 5) könnte das Endergebnis wie folgt aussehen: Nach der Berechnung des Bestandes dieses Flurförderzeugtyps werden die Verbrauchsangaben mit der erwarteten Betriebsstundenzahl pro Jahr multipliziert. Unter den getroffenen Annahmen ergibt sich in der Europäischen Union (EU-27) für Dreirad-Elektro-Gegengewichtsstapler ein Gesamtenergieverbrauch von minimal 1,9 TWh und maximal 2,9 TWh pro Jahr.

Eine Zahl soll zum Vergleich herangezogen werden: Der Google-Konzern hatte im Jahr 2010 ungefähr 2,26 TWh verbraucht; das sei laut [Süd12] so viel, wie eine 
US-Stadt mit 100.000 bis 200.000 Einwohnern pro Jahr verbrauche.

Zum Zweck der Vergleichbarkeit werden zusätzlich weitere Umweltaspekte, wie z. B. $\mathrm{CO}_{2}$-Emissionen bzw. $\mathrm{CO}_{2}$-äquivalente Emissionen berechnet. Um stetig weiter verbessert werden zu können, soll das Modell ferner jegliche Anpassung der in ihm getroffenen Annahmen ermöglichen. Dadurch ließen sich z. B. problemlos der VDIVerbrauch oder einbezogene Ladefaktoren variieren.

\section{TEILPROJEKT KRANE UND HEBEZEUGE (FML)}

\subsection{Produktdefinition}

Als klassischer Unstetigförderer, der seine Hauptarbeitsbewegung im Aussetzbetrieb ausführt [Sch94], werden Krane für vielfältige Hub- und Transportaufgaben in der Industrie und im Bauwesen eingesetzt. Durch die diversen Aufgaben existieren eine ganze Reihe unterschiedlicher Kranbauarten [DIN73], z. B. Brückenkrane, Turmdrehkrane oder Säulen- bzw. Wandschwenkkrane. Da für die Ermittlung der Umweltaspekte auf europäischer Ebene der aktuelle Kranbestand berechnet werden muss, wird im Rahmen der Untersuchung auf eine Kategorisierung in Anlehnung an die europäische Produktionsstatistik Prodcom [EWG91] zurückgegriffen. Die vier maßgeblichen Kranbauarten, die innerhalb des Forschungsprojekts betrachtet werden, sind in Abbildung 7 dargestellt.

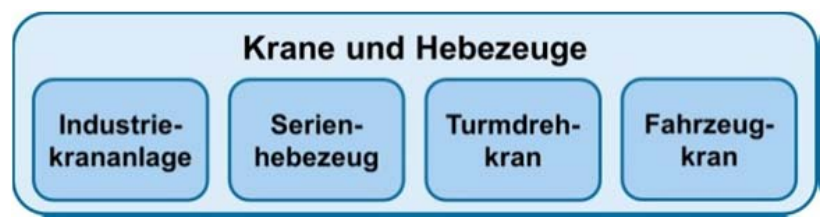

Abbildung 7. Untersuchte Kranbauarten

Unter dem Begriff Industriekrananlagen werden die Prodcom-Kategorien Brückenkrane und Konsolkrane zusammengefasst, da beide Kranbauarten einen ähnlichen Einsatzbereich besitzen und einen quaderförmigen $\mathrm{Ar}$ beitsbereich abdecken. Die Serienhebezeuge umfassen alle Produkte, die als elektrische Flaschenzüge und Kettenzüge an die Prodcom-Statistik gemeldet werden. Nach [Gol04] sind bei diesen Bauarten meist polumschaltbare Motoren als Antriebe verbaut. Turmdrehkrane sind in der Ausführung als Oben- oder Untendreher für Hubaufgaben auf Baustellen zu finden, wobei die Auswahl und die Verwendung des Krans von einer Vielzahl von Faktoren abhängig sind und eine exakte Planung voraussetzten [Kön11]. Als Referenz für den Fahrzeugkran wird innerhalb des Forschungsprojektes der All-Terrain-Kran verwendet, dessen Fahrantrieb sowohl für den Straßenverkehr als auch für den Einsatz im Gelände geeignet ist und den Kran damit zu einer äußerst universell einsetzbaren Maschine macht [Kön11].
Im vorliegenden Beitrag wird am Beispiel der Industriekrananlage eine Vorgehensweise vorgestellt, wie die Umweltaspekte beim Betrieb von Kranen auf Bestandsebene in der Nutzungsphase quantifiziert und bewertet werden können.

\subsection{BESTANDSERMITTLUNG UND VERWENDUNG VON KRANANLAGEN}

Im Rahmen der Quantifizierung und Erfassung der relevanten Umweltaspekte von Industriekrananlagen muss in einem ersten Schritt die Population bestimmt werden. Dazu werden die jährlichen Verkaufszahlen aus der Prodcom-Statistik mit den Import- und Exportzahlen aus der europäischen Außenhandelsstatistik [Eur12a] verrechnet und daraus ein jährlich neu aufgebauter Bestand ermittelt. Durch das Aufsummieren des jedes Jahr neu aufgebauten Bestandes über die geschätzte Lebensdauer von 20 Jahren [Ber89] wird der augenblicklich in der EU installierte Bestand abgeschätzt [Kem12]. Für die Industriekrananlage ergibt sich nach diesem Vorgehen ein aktueller Bestand von ca. 800.000 Einheiten in der EU.

Im weiteren Verlauf der Quantifizierung muss geklärt werden, wie die betreffenden Krananlagen eingesetzt werden, d. h. welche Lasten in welcher Häufigkeit vom Kran transportiert werden. An dieser Stelle erweisen sich die standardisierten Lastkollektive nach der FEM-Richtlinie 9.511 [FEM86] als praktikabler Ansatzpunkt. Diese zeitbezogenen Lastkollektive (siehe Abbildung 8) geben die relative Größe der Lasten mit den entsprechenden relativen Zeitanteilen an, mit denen das Hubwerk eines Krans beansprucht wird. In [Jod12] wird bei der Auswahl von repräsentativen Betriebsparametern für Energiebedarfsmessungen ebenfalls diese Möglichkeit diskutiert.

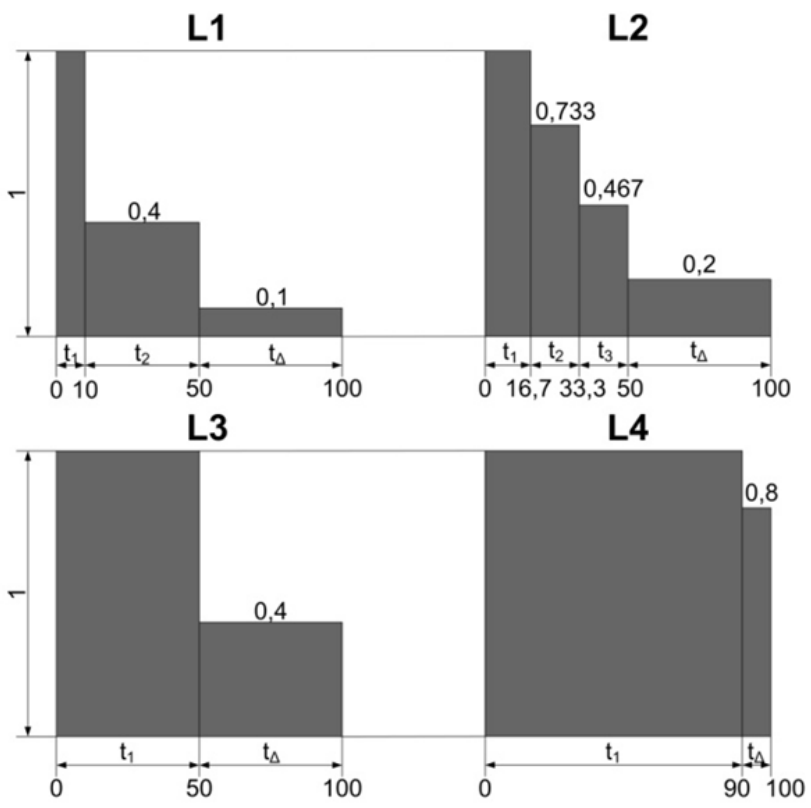

Abbildung 8. Lastkollektive nach [FEM 9.511] 
Diese zeitbezogenen Kollektive werden mit Hilfe der ebenfalls in [FEM86] angegebenen Volllastlebensdauern in arbeitsspielbezogene Kollektive, wie sie in der europäischen Krannorm [EN09] üblich sind, überführt. In [Gol04] wird dieser Weg sehr ausführlich dargestellt. Die Dauer eines jeden Spiels ergibt sich aus der [FEM95] zu 0,6 Minuten für den Aussetzbetrieb und wird paritätisch auf den Hub- und den Senkvorgang verteilt. Das gleiche Vorgehen gilt analog auch für die Antriebe der Kranbrücke und der Laufkatze.

\subsection{Messungen}

Als Basis für die Ermittlung der Umweltaspekte des Energiebedarfs werden an verschiedenen Brückenkranen Leistungsaufnahmemessungen durchgeführt. Die Messungen werden mit Hilfe von geeichten Prüflasten bei der jeweils größten möglichen Hubgeschwindigkeit durchgeführt (siehe Abbildung 9). Die Leistungsaufnahmemessungen werden direkt im Schaltschrank mit einem Netzanalysator durchgeführt, um Spannung, Stromstärke und die aufgenommene Leistung aufzeichnen zu können.

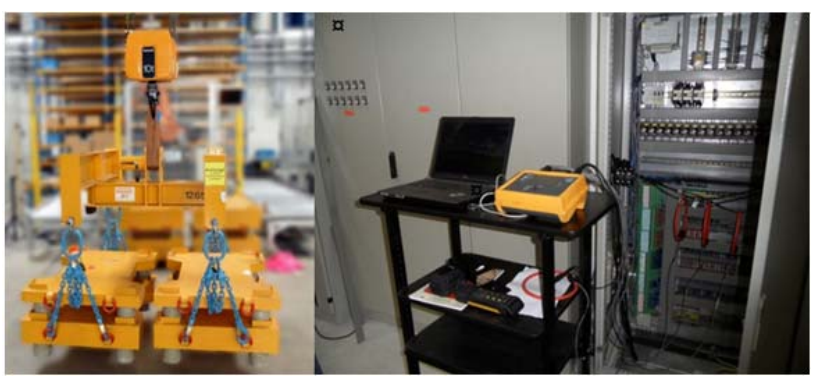

Abbildung 9. Leistungsaufnahmemessungen

Um aus der gemessenen Leistung Rückschlüsse auf die Wirkungsgrade des Hubwerkes zu ziehen, wird mit einem Seilzugwegsensor die Hubgeschwindigkeit aufgenommen und aus diesen Messwerten der Wirkungsgrad des Hubwerkes unter dem Einfluss verschieden großer Hublasten berechnet. Unter den Annahmen, dass die Wirkungsgrade sowohl von Seilrollen und -trommeln [Sch94] als auch von Zahnrädern bei hinreichend großer Belastung [Mül98] von den belastungsabhängigen Verlustanteilen dominiert werden, wird aus dem Gesamtwirkungsgrad auf den Motorwirkungsgrad rückgerechnet. Damit stehen an dieser Stelle alle Informationen zur Verfügung, um im nächsten Schritt mit Hilfe eines Simulationsmodells den Energiebedarf des Krans unter verschiedenen Szenarien berechnen zu können.

\subsection{SIMULATIONEN UND HOCHRECHNUNGEN}

Die Möglichkeit, Energiebedarfe für Fördermittel in der Intralogistik durch ein Modell in Matlab/ Simulink zu berechnen wurde in [Ert12] für Regalbediengeräte bereits vorgestellt. Für Brückenkrane wird ein parametrisches Simulationsmodell entwickelt, mit dem der Energiebedarf während der Nutzungsdauer eines Krans in Abhängigkeit von seinen Betriebsparametern abgebildet werden kann.
Die aus den Messungen gewonnen Erkenntnisse dienen als Datenbasis für das Modell. Abbildung 10 zeigt exemplarisch den Vergleich einer Messung mit der dazugehörigen Simulation bei einem polumschaltbaren Antrieb. Das Anlaufverhalten des Motors wird innerhalb des Modells mit der Tangens-Hyperbolicus-Funktion abgebildet [Gol04].

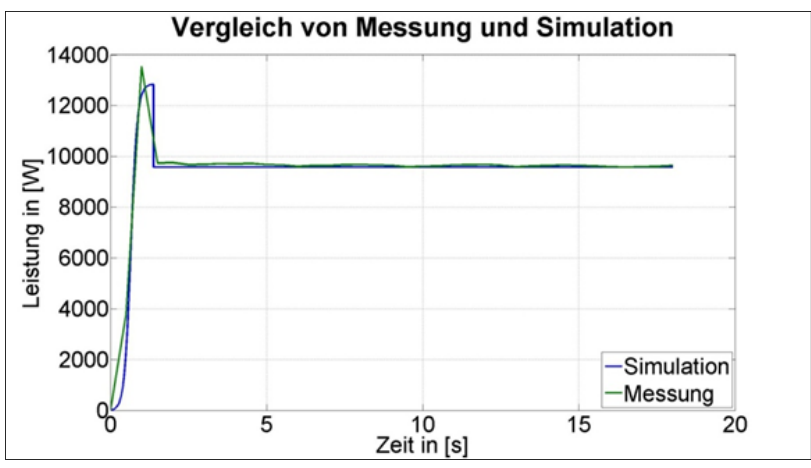

Abbildung 10. Vergleich zwischen Messung und Simulation für einen Hubwagen

Die Verluste in den einzelnen Baugruppen des Hubwerkes werden mit Hilfe von Wirkungsgraden abgebildet, dabei wird die Lastabhängigkeit des Motorwirkungsgrades ebenfalls berücksichtigt. Mit diesem Modell steht ein Werkzeug zu Verfügung, den Energiebedarf von Brückenkranen mit hinreichender Genauigkeit vorherzusagen. Bei den Simulationen wird immer nur die größte mögliche Hubgeschwindigkeit berücksichtigt, um die obere Grenze des Energiebedarfs abzuschätzen.

Abbildung 11 zeigt die Abhängigkeit des Energiebedarfs von der Einstufung des Hubwerkes und vom Lastkollektiv. Zugrunde liegt diesen Berechnungen eine Lebensdauer des Hubwerkes von 20 Jahren, in denen die Volllaststunden nach [FEM86] erreicht werden. Die Abhängigkeit des Energiebedarfs von der Einstufung ergibt sich zum einen aus der steigenden Anzahl der Spiele pro Stunde und zum anderen aus der größeren Anzahl der Volllaststunden. Die Vergrößerung des Energiebedarfs hin zu kleineren Lastkollektiven ergibt sich aus der Tatsache, dass die tatsächliche Einschaltzeit im betreffenden Lastkollektiv bei kleineren Kollektiven deutlich zunimmt, bis die dieser Beanspruchung äquivalente Volllastlebensdauer erreicht ist. Berechnet werden die dargestellten Ergebnisse mit Hilfe des Matlab/ Simulink-Modells, wobei als Referenz ein fiktives 10t Hubwerk mit polumschaltbaren Antrieben Verwendung findet. 


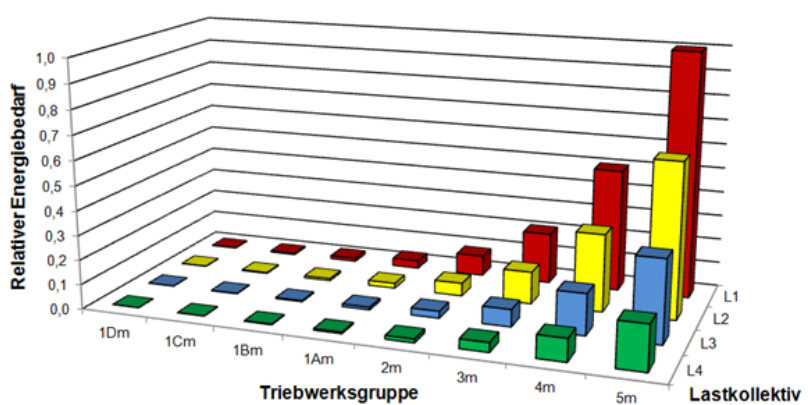

Abbildung 11. Energiebedarf in Abhängigkeit des Lastkollektivs und der Einstufung des Hubwerkes

\subsection{ENERGIEBEDARF UND ABLEITUNG WEITERER UMWELTASPEKTE}

Mit den Daten zur Verwendung des Krans und dem Simulationsmodell kann der Gesamtenergiebedarf eines Krans während seiner Nutzungsdauer berechnet werden. Aus diesem Energiebedarf werden im weiteren Verlauf die betrachteten Umweltaspekte abgeleitet. Das Vorgehen hierzu ist in Abbildung 12 veranschaulicht.

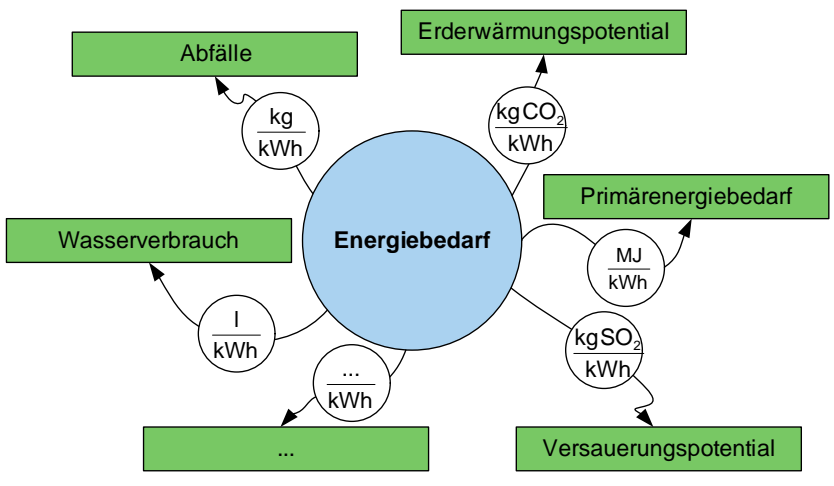

Abbildung 12. Ableitung der Umweltaspekte aus dem Energiebedarf

Für die Ermittlung der Umweltaspekte werden Einheitswerte aus öffentlich zugänglichen Quellen wie dem EcoReport [Kem12] oder der Probas-Liste [Öko12] verwendet. Zusätzlich kann durch die Auswahl eines mittleren Referenzkrans und dem berechneten Bestand der Gesamtenergiebedarf bzw. die Umweltaspekte der Industriekranpopulation in Europa abgeschätzt werden.

Wählt man als Referenz für den Bestand an Industriekranen eine Anlage mit den in Tabelle 2 dargestellten Werten und berechnet daraus den jährlichen Energiebedarf für den Bestand, so erhält man nach dieser Vorgehensweise einen Bedarf von ca. 0,8 Terawattstunden (TWh) elektrischer Energie jährlich. Vergleicht man diese Zahl mit dem in der europäischen Union auftretenden Gesamtbedarf an elektrischer Energie von ca. 2.850 TWh im Jahr 2010 [Eur12b], so ergibt sich nur ein relativ kleiner Anteil des Energiebedarfs, der auf diese Kranbauart zurückzuführen ist.
Tabelle 2. Daten Referenzkrananlage

\begin{tabular}{|c|c|}
\hline Tragfähigkeit & $10 \mathrm{t}$ \\
\hline Einstufung Hubwerk: & $2 \mathrm{~m}$ \\
\hline Lastkollektiv: & $\mathrm{L} 1$ \\
\hline Hubgeschwindigkeit: & $6 \mathrm{~m} / \mathrm{min}$ \\
\hline
\end{tabular}

\subsection{ZUSAMMENFASSUNG}

Im Rahmen dieses Beitrags wird eine Möglichkeit vorgestellt, den Energiebedarf und die daraus abgeleiteten Umweltaspekte für Brückenkrananlagen zu bestimmen. Durch den Bezug auf die gängigen Auslegungsnormen für Hebezeuge und Kranantriebe wird die Art der Verwendung des Krans dargestellt. Mit Hilfe von Leistungsaufnahmemessungen an existierenden Krananlagen können Rückschlüsse auf Wirkungsgrade verschiedener Hubwerke gezogen werden. Mit diesen Informationen wird ein Simulationsmodell erstellt, welches es ermöglicht, den Energiebedarf eines Brückenkrans in Abhängigkeit von seinen Betriebsparametern zu bestimmen. Nach der Abschätzung des Bestandes wird der Energiebedarf für die Population dieser Kranbauart in der EU-27 zu ca. 0,8 TWh berechnet. Aus diesem Energiebedarf können die weiteren Umweltaspekte mit Hilfe von bekannten Einheitswerten abgeleitet werden.

\section{TEILPROJEKT LAGERTECHNIK (IFL)}

\subsection{SYSTEMGRENZEN: LAGER- UND KOMMISSIONIERVORGANG}

Ein wichtiger Faktor im Wettbewerb der Unternehmen ist es die geeignetste Verteilung ihrer produzierten Waren auf dem Absatzmarkt sicherzustellen. Eine Möglichkeit dafür stellt der Betrieb eines Verteilzentrums, eines sogenannten Distributionszentrums, dar. Ein Distributionszentrum beinhaltet in der Regel die Prozesse „Wareneingang“, „Lagern und Kommissionieren“, „Konsolidieren und Verpacken“, „Warenausgang“ und ggf. „Added Value“. Besondere Bedeutung erhält der Prozess „Lagern und Kommissionieren“, da bis zu 63 \% der Gesamtkosten dort anfallen sowie $55 \%$ des Personals eines Distributionszentrums dort arbeiten [Mal91; LeD05]. Demnach ist der Lager- und Kommissioniervorgang in fast jedem Distributionszentrum der arbeitsintensivste und teuerste Prozess [DLR06]. Außerdem stellt er die schwierigste Aufgabe der innerbetrieblichen Logistik dar [Gud05].

Im Lager- und Kommissioniervorgang können die Aufgaben durch viele unterschiedlichste Fördermittel der Intralogistik ausgeführt werden. So kann ein Transport entweder über eine fest installierte Förderstrecke, z. B. einen Rollenförderer, erfolgen, oder mit Hilfe von Unstetigförderern, wie z. B. fahrerlosen Transportsystemen, ermöglicht werden. 
Um diesen arbeitsintensiven und teuren Vorgang auch anhand ökologischer Faktoren evaluieren zu können, kann mit Hilfe der Betrachtung der Umweltaspekte und -auswirkungen eine erste Bewertung vorgenommen werden. Ein Ansatz zur ganzheitlichen Betrachtung der Umweltaspekte des Lager- und Kommissioniervorgangs ist die gegenseitige Beeinflussung der einzelnen Fördermittel im Lager- und Kommissioniervorgang mit zu berücksichtigen.

Der Lager- und Kommissioniervorgang wird deshalb anhand des Gesamtsystems „Technik im Lager- und
Kommissioniervorgang“ betrachtet. Dieses Gesamtsystem kann in verschiedene Teilsysteme unterteilt werden, z. B. in das Teilsystem „Fördertechnik“ oder „Lagertechnik“. Das Teilsystem „Lagertechnik“ besteht dabei aus weiteren Teilsystemen, z. B. dem Teilsystem „Regalbediengerät“. In Abbildung 13 ist schematisch die Aufteilung der „Technik im Lager- und Kommissioniervorgang“ in Systeme bzw. Teilsysteme dargestellt.

\section{Technik im Lager- und Kommissioniervorgang}

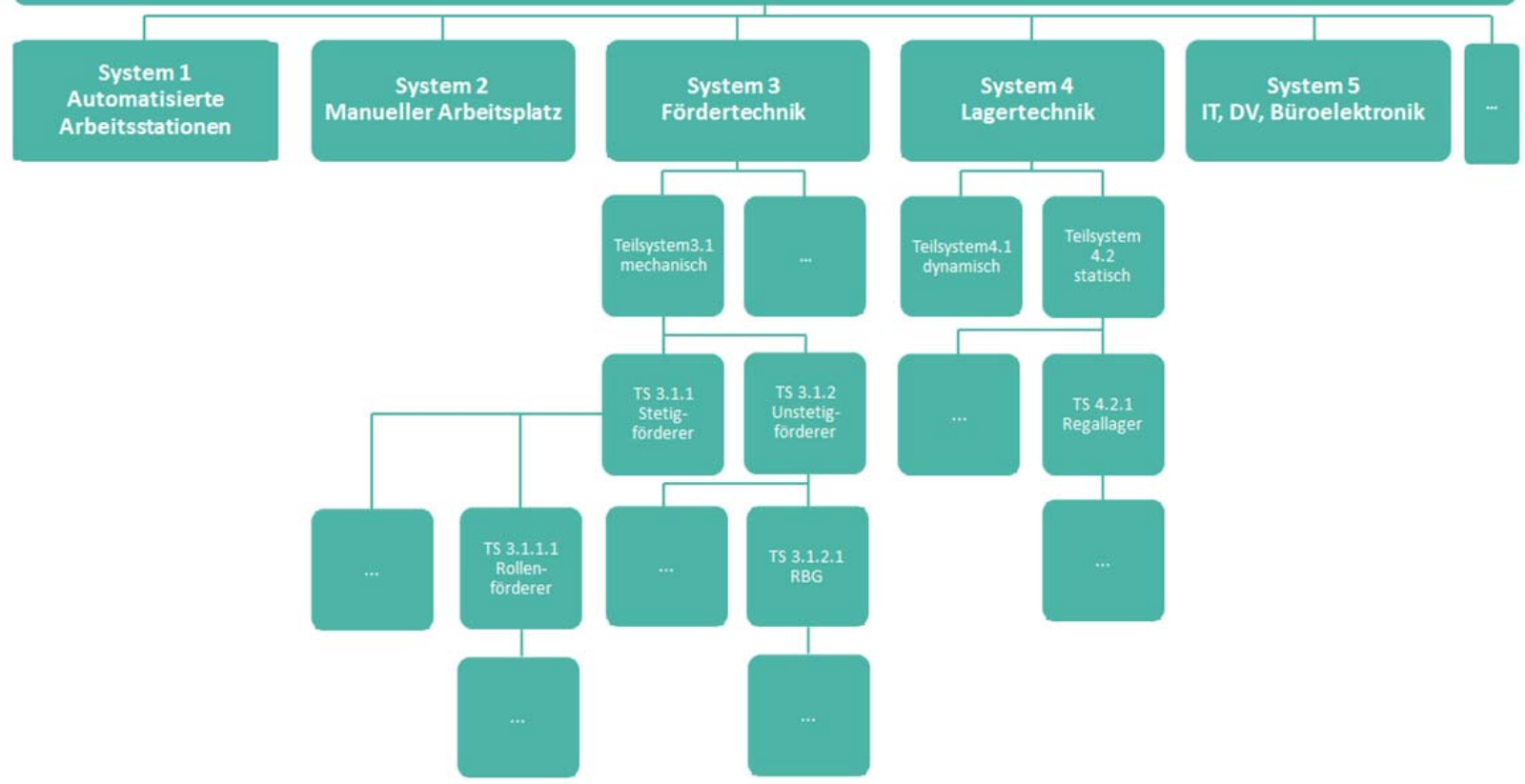

Abbildung 13. Darstellung der Systembetrachtung der Technik im Lager- und Kommissioniervorgang

Folgendes Beispiel soll die grundlegenden Überlegungen der Systembetrachtung im Lager- und Kommissioniervorgang verdeutlichen: Die Aufgabe „Einlagern, Auslagern und Kommissionieren“ kann z. B. über zwei unterschiedliche Vorgehensweisen erfolgen. So kann ein Artikel auf einer Palette im Hochregallager gelagert und in mehreren Schritten von der Palette kommissioniert werden, andererseits kann zunächst im Wareneingang die Palette auf Kleinladungsträger vereinzelt und im AKL/ Shuttlelager gelagert und daraus kommissioniert werden. Die beschriebenen unterschiedlichen Abläufe stellen einen Unterschied bei der Betrachtung z. B. des Energiebedarfs oder des Leistungsverlaufs dar.

Aufgrund dieser Systemüberlegungen wird im Projekt Lagertechnik die Systemgrenze nicht um ein einzelnes Gerät, sondern um den kompletten Vorgang gelegt.
Ein Rückschluss aus der Betrachtung der einzelnen Fördermittel auf die Umweltauswirkungen des gesamten Lager- und Kommissioniervorgangs wird durch den modularen Aufbau intralogistischer Vorgänge und Materialflussprozesse mit Hilfe des Systemgedankens ermöglicht.

\subsection{UMWELTASPEKTE UND UMWELTAUSWIRKUNGEN IM LAGER- UND KOMMISSIONIERVORGANG}

Eine vollständige Übersicht aller Umweltaspekte von Fördermitteln in der Intralogistik ist innerhalb des Projektes erarbeitet worden. Ziel dabei ist die Identifikation, Quantifizierung, Analyse und Bewertung der Umweltauswirkungen einzelner Fördermittel entlang deren vollständigen Produktlebenszyklus. Diese Betrachtung über den vollständigen Lebenszyklus lässt eine Bewertung der Beeinflussbarkeit der Umweltaspekte durch die jeweiligen Lebensphasen zu. 
Eine Möglichkeit, die Umweltaspekte auch über den Lebenszyklus zu quantifizieren und vereinfachte Aussagen über Umweltauswirkungen $\mathrm{zu}$ treffen, liefert das EcoReport-Tool [Kem12] der Europäischen Kommission, das im Rahmen der MEEuP-Methode [Kem12] der Ökodesign-Richtlinie entwickelt wurde. Durch Angabe der verbauten Werkstoffe, deren Massen und weiterer Daten zu einzelnen Lebensphasen können Umweltauswirkungen von energiebetriebenen Produkten abgeschätzt werden.

Abbildung 14 stellt Ergebnisse des EcoReport-Tools am Beispiel eines Paletten-Regalbediengerätes dar. Dabei sind die Höhe einiger Umweltaspekte, z. B. Triebhausgase (im GWP100), flüchtige organische Verbindungen (VOC), oder langlebige organische Schadstoffe (POP), prozentual über alle Lebensphasen der Ökodesignrichtlinie [EU05] angegeben.

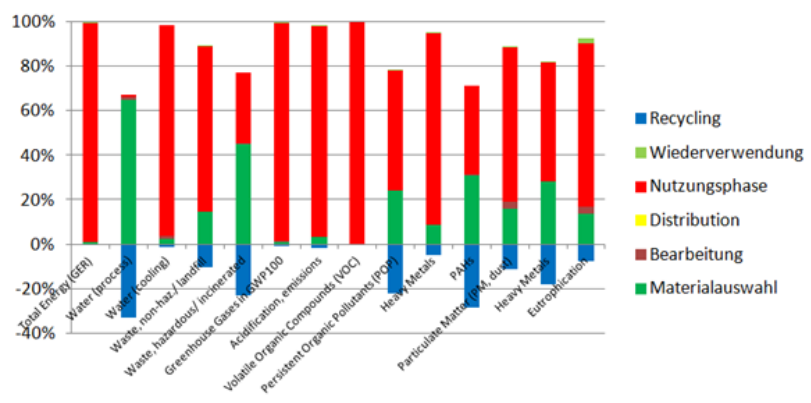

Abbildung 14. Darstellung der Ergebnisse des Eco-ReportTools am Beispiel eines Paletten-RBGs über die Lebensphasen (in Anlehnung an [Sch12])

Aus den Ergebnissen wird deutlich, dass die Umweltaspekte durch die Nutzungsphase am meisten beeinflusst werden. Mit Hilfe des EcoReport-Tools ist allerdings eine genaue Betrachtung der Nutzungsphase nicht möglich, da dort nur eine Aussage über den Gesamtenergiebedarf getroffen wird. Deshalb sind im Teilprojekt Lagertechnik eigenständige analytische Modelle eines jeden Fördermittels (Teilsystems) im Lager- und Kommissioniervorgang speziell für die Nutzungsphase erstellt worden. Durch Zusammenfügen dieser einzelnen Modelle kann z. B. der Energiebedarf und Leistungsverlauf der unterschiedlichen technischen Umsetzungen der intralogistischen Vorgänge und Ausprägungen der Materialflussprozesse angegeben werden. Deshalb werden innerhalb des Projektes zehn verschiedene Umweltaspekt-Teilsystemmodelle für Fördermittel, die an den Prozessen Transport, Einlagern, Lagern und der Entnahme [Wis09] beteiligt sind, erstellt.

Grundlage dieser Modelle ist der Leistungsverlauf $\mathrm{P}(\mathrm{t})$, anhand dessen der Gesamtenergiebedarf errechnet werden kann. Ausgehend vom Energiebedarf können auf Basis eines Stromerzeugungsmixes [Öko12] über bekannte Umrechnungskennzahlen mehrere Umweltaspekte, z. B. Luftemissionen (wie der $\mathrm{CO}_{2}$-Ausstoß) speziell für die Nutzungsphase, berechnet werden.
Ziel der Teilsystem-Modelle ist es neben der Analyse z. B. der Leistungsverläufe oder der Betrachtung des Gesamtenergiebedarfs, eine Bewertung der unterschiedlichen Energieeffizienzmaßnahmen in der Nutzungsphase zu ermöglichen. Durch Eingabe charakteristischer Größen wie der Geschwindigkeit, der Beschleunigung, der Massen oder der Verlustgrößen in die Modelle kann der Gesamtenergiebedarf und der Leistungsverlauf eines Fördermittels für eine bestimmte Tätigkeit im Lager- und Kommissioniervorgang angegeben werden.

Dabei sind die Modelle nicht auf eine Geräteklasse beschränkt, sondern können dem jeweiligen Gerät individuell für verschiedene Aufgaben angepasst werden. Neben der Abbildung des Stands der Technik heutiger Systeme, kann mit Hilfe der Modelle auch das Energieeinsparpotential neuer Technologien und der Einfluss verschiedener Abläufe abgeschätzt werden. Darüber hinaus lässt sich anhand der Modelle die Spielzeit, d. h. die Zeit, die zum Ausführen eines Arbeitsspiels benötigt wird, angeben.

Abbildung 15 zeigt einen Leistungsverlauf aus dem Modell „Regalbediengerät“. Zur Verifizierung aller Modelle und zur Ermittlung fehlender Parameter, wie z. B. der (Fahr-) Widerstandsbeiwerte, werden Leistungsmessungen an realen Systemen durchgeführt (siehe Abbildung 16).

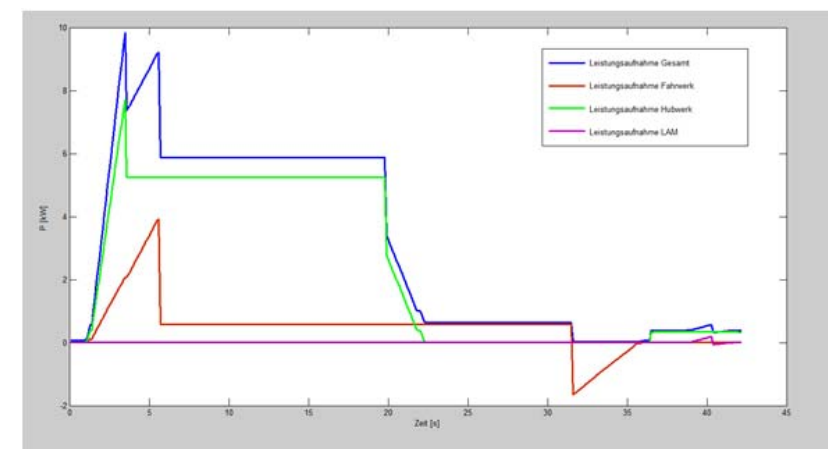

Abbildung 15. Leistungsverlauf eines Paletten-Regalbediengerätes über eine Einzelfahrt vom E/A (0/0/0) zum Fach (60/20/1)

Die Kombination aus EcoReport-Tool und den analytischen Energiebedarfsmodellen der Nutzungsphase ermöglicht damit sowohl die Darstellung einer Übersicht der Umweltaspekte über alle Lebensphasen, als auch eine Analyse der Höhe der Umweltaspekte der einzelnen Übertragungsglieder in der Nutzungsphase. 


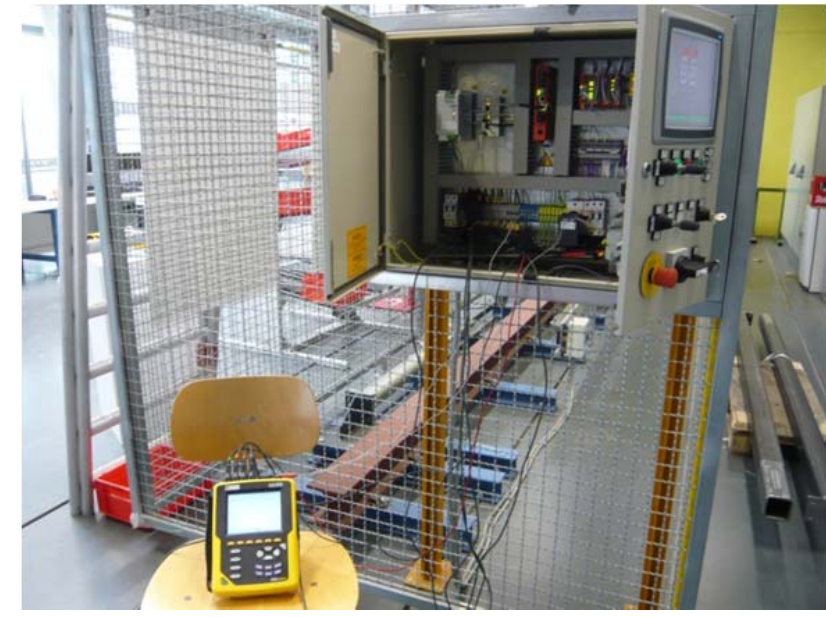

Abbildung 16. Leistungsmessung des Regalbediengeräts auf der Versuchsfläche des IFL

\subsection{ZUSAMMENFASSUNG UND AUSBLICK}

Im Teilprojekt Lagertechnik wird die Systemgrenze nicht um ein einzelnes Fördermittel, sondern um den kompletten Lager- und Kommissioniervorgang gelegt, da die Aufgaben innerhalb dieses Vorgangs durch eine Vielzahl technischer Fördermittel realisiert werden können. Aufbauend auf dieser Systembetrachtung wurde in einem ersten Schritt der Lager- und Kommissioniervorgang in unterschiedliche Teilsysteme aufgeteilt. Um eine ökologische Bewertung des Vorgangs zu realisieren, wird die Höhe der Umweltaspekte zum einen über das EcoReportTool der Europäischen Kommission über alle Lebensphasen, sowie zum anderen über eigenständige analytische Umweltaspekt-Modelle der Fördermittel im Lager- und Kommissioniervorgang in der Nutzungsphase quantifiziert. Diese Kombination ermöglicht eine ganzheitliche Betrachtung der Umweltaspekte der Fördermittel im Lager- und Kommissioniervorgang über alle Lebensphasen.

Es wird deutlich, dass die Betrachtung aller Umweltaspekte im Lager- und Kommissioniervorgang ein herausforderndes Thema für Industrie und Forschung ist. Neben der Entwicklung analytischer Modelle sollten auch standardisierte Möglichkeiten zur Bewertung dieser Systeme, z. B. über Kennzahlen, entwickelt werden. Damit sollen unterschiedliche Intralogistiksysteme für Anwender und Anbieter dieser Systeme bewertet werden. Ein Ansatz zur Bewertung unterschiedlicher Fördermittel hinsichtlich ihrer Umweltaspekte wurde im Projekt ebenfalls erarbeitet.

\section{KENNGRÖßEN ZUR BEWERTUNG DER UMWELTLEISTUNG}

Ein Schwerpunkt der Betrachtung in diesem Projekt ist die Bestimmung der Umweltaspekte anhand des Energiebedarfs der Fördermittel in der Nutzungsphase. Eine Möglichkeit zur Bewertung besteht darin, in einer Kennzahl den Energiebedarf einer erbrachten Leistung gegen- über zu stellen. Dabei wird angestrebt, eine Definition der Logistikleistung zu erarbeiten, die unabhängig vom Fördermittel Gültigkeit besitzt.

Im Allgemeinen dienen Kennzahlen und Kenngrößen zur Quantifizierung und Bewertung von Sachverhalten und stellen damit die Grundlage für Vergleiche dar. Eine Kenngröße besteht dabei aus einer Maßzahl und der dazugehörigen Einheit. Im Rahmen von Umweltmanagementsystemen ist eine Kennzahl die „messbare Darstellung des Zustands oder Status von Leistung, Management, oder Bedingungen“ [DIN12].

Ein grundsätzliches Unterscheidungsmerkmal von Kennzahlen besteht darin, ob es sich um eine relative oder eine absolute Kennzahl handelt. Im Fall relativer Kenngrößen wird die Aussage ins Verhältnis zu einer Bezugsgröße gesetzt. Bei Kenngrößen zur Effizienz ist es immer das Ziel, den Ertrag in Bezug auf die Aufwendungen zu stellen. So wird Energieeffizienz in [EU06] als „das Verhältnis von Ertrag an Leistung, Dienstleistungen, Waren oder Energie zum Energieeinsatz“ definiert. Ein Beispiel für eine relative Kenngröße ist der Kohlenstoffdioxidausstoß eines Lastkraftwagens (LKW) bezogen auf seine Transportleistung in Tonnenkilometern $\left(\mathrm{CO}_{2} / \mathrm{tkm}\right)$. Mit dieser Kenngröße ist es nicht nur möglich, Vergleiche von LKW durchzuführen, sondern auch den LKW mit anderen Fördermitteln wie der Bahn oder dem Schiffstransport zu vergleichen. Allen diesen Vergleichen liegt die Tatsache zu Grunde, dass die Fördermittel sich entlang einer Strecke bewegen. Im Unterschied dazu muss bei der Bewertung der Umweltleistung von Intralogistiksystemen der Hubvorgang, der bei vielen Unstetigförderern wie Flurförderzeugen, Kranen oder Regalbediengeräten auftritt, besondere Berücksichtigung finden.

Für die energetische Bewertung intralogistischer Systeme werden in [Jod12] erste Ansätze geliefert. Hier wird das Konzept der Bildung einer Energieeffizienzkennzahl aufgegriffen und an einem Rollenförderer angewendet. Um den Energieeinsatz bestimmen zu können, werden verschiedene Messpunkte an den Maschinen definiert und die Energiebedarfe im Strip-Down-Verfahren an diesen Messstellen bestimmt. Bei der Vorgabe eines definierten Lastkollektivs ergibt sich die Kenngröße als Transportweg in Bezug zu dem benötigten Energieeinsatz.

Ein anderer Ansatzpunkt zur Bewertung des Energiebedarfs in intralogistischen Systemen kann aus [DIN12] abgeleitet werden. Auf Grundlage der In- und Outputs des Systems wird eine Kenngröße abgeleitet, die den Energiebedarf in Relation zu einer Logistikleistung setzt. Abbildung 17 veranschaulicht dieses Vorgehen. 


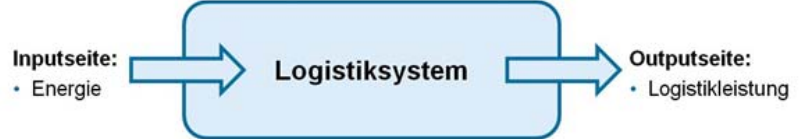

Abbildung 17. Betrachtung eines Logistiksystems

Eine Möglichkeit, um die Umweltaspekte oder den Energiebedarf eines Logistiksystems zu bewerten, ist die die Definition einer Logistikleistung. Diese Größe bildet die Vielzahl von Anforderungen an das Logistiksystem ab. Anforderungen an ein Logistiksystem sind beispielsweise der Durchsatz des Systems oder die transportierten Massen. Die Kenngröße wird damit aus dem Quotienten des Energiebedarfs und der Logistikleistung gebildet und der Bedarf an Energie damit bewertet.

\section{ZUSAMMENFASSUNG}

Im Rahmen dieses Beitrags wird ein Überblick über die Analyse, Quantifizierung und Bewertung der Umweltaspekte von Fördermitteln in der Intralogistik gegeben. Dazu werden die einzelnen Lebensphasen der Produkte hinsichtlich der Höhe ihrer Umweltaspekte analysiert und ausgewertet. Grundlegende Unterschiede ergeben sich in den Teilprojekten durch die Wahl der Systemgrenze. Während im Fall von Kranen und Hebezeugen sowie den Flurförderzeugen nur die Maschine an sich betrachtet wird, erfolgt in der Lagertechnik eine Betrachtung des gesamten Lager- und Kommissioniervorgangs mit seinen Wechselwirkungen. Innerhalb der Nutzungsphase werden Messungen und Modelle verwendet, um den Energiebedarf der Geräte zu erfassen und daraus Rückschlüsse auf weitere Umweltaspekte zu ziehen. Zur Bewertung der Umweltaspekte wird ein Vorgehen skizziert, den Energiebedarf als einen zentralen Aspekt in Bezug zur geleisteten Arbeit zu setzen. Die geleistete Arbeit ergibt sich dabei aus den Ausgangsgrößen, die im System enthalten sind.

\section{LITERATUR}

[Ber89] von Berg, Dietrich: Krane und Kranbahnen. Teubner, Stuttgart, 1989

[Bun12] Bundesministerium der Finanzen: $A b$ schreibungstabelle für allgemein verwendbare Anlagegüter, http://www. bundesfinanzmi-

nisterum.de/Content/DE/Standardartike 1/Themen/Steuern/Weitere_Steuertheme n/Betriebspruefung/AfA-Tabellen/afatabellen-anl.pdf?__blob

=publicationFile $\& \mathrm{v}=4$, am 02.04.2012

[DIN73] DIN 15001:1973: Krane - Begriffe, Einteilung nach der Bauart, Beuth, Berlin, 1973
[DIN94]

[DIN06]

[DIN09]

[DIN12]

[DLR06]

[EN09]

[Ert12]

[EU05]

[EU06]

[Eur12a]

Eurostat: Datenbank internationaler Handel, http://epp.eurostat.ec.europa .eu/portal/page/portal/international_trad e/data/database; Aufgerufen am 03.08.2012

DIN ISO 5053:1994: Kraftbetriebene Flurförderzeuge, Beuth, Berlin, 1994

DIN EN ISO 14044:2006: Umweltmanagement - Ökobilanz - Anforderungen und Anleitungen, Beuth, Berlin, 2006

DIN EN ISO 14001:2009: Umweltmanagementsysteme - Anforderungen mit Anleitung zur Anwendung, Beuth, Berlin, 2009

DIN EN ISO 14031: Umweltmanagement - Umweltleistungsbewertung Leitlinien, Beuth, Berlin, 2012

De Koster, René; Le-Duc, Tho; Roodbergen, Kees: Design and control of warehouse order picking: a literature review. ERIM Report Series Research in Management ERS-2006-005-LIS, 2006

EN 13001-1:2009: Krane - Konstruktion allgemein - Teil 1: Allgemeine Prinzipien und Anforderungen, Beuth, Berlin, 2009

Ertl, Rainer; Günthner, Willibald A.; Fischer, Gabriel; Hahn-Woernle, Paul: Energieeffiziente Intralogistik auf $\mathrm{Ge}$ räteebene - Beispiel Regalbediengerät In: Schenk, Michael; Zadek, Hartmut; Müller, Gerhard; Richter, Klaus; Seidel, Holger (Hrsg.): Tagungsband 17. Magdeburger Logistiktage, Sichere und Nachhaltige Logistik, Otto-vonGuericke-Universität Magdeburg, Magdeburg, 27.06.2012

Europäische Kommission: Richtlinie 2005/32/EG zur Schaffung eines Rahmens für die Festlegung von Anforderungen an die umweltgerechte Gestaltung energiebetriebener Produkte, Straßburg, 2005

Europäische Kommission: Richtlinie 2006/32/EG über Endenergieeffizienz und Energiedienstleistungen, Straßburg, 2006

03.08 .2012 
[Eur12b] Eurostat: Energieendverbrauch von Elektrizität http://epp.eurostat.ec.europa .eu/portal/page/portal/energy/data/main _tabtab; Aufgerufen am 03.08.2012

[EWG91] EWG-Verordnung 3924/91 des Rates zur Einführung einer Gemeinschaftserhebung über die Produktion von Gütern, Brüssel, Europäischer Rat, 1991

[FEM86] FEM-Richtlinie 9.511: Berechnungsgrundlage für Serienhebezeuge: Einstufung der Triebwerke, VDMA-Verlag, Frankfurt am Main, 1986

[FEM95] FEM - Richtlinie 9.683: Serienhebezeuge - Auswahl von Hub- und Fahrmotoren, VDMA-Verlag, Frankfurt, 1995

[Gol04] Golder, Markus: Ein Beitrag zur Kostenabschätzung von für Brückenkranträger in Kastenbauweise auf Basis ihrer Bemessungsgrundlagen und Dimensionierungsnachweise, Dissertation, Technische Hochschule Karlsruhe, 2004

[Gud05] Gudehus, Timm: Logistik - Grundlagen, Strategien, Anwendungen, 3. Neu beabeitetete Aufl. , Berlin, Springer Verlag 2005, S. 553

[Gün09] Günthner, Willibald A.; Seebauer, Petra; Boppert, Julia; Tenerowicz, Peter: Studie „Change to Green - Handlungsfelder und Perspektiven für nachhaltige Logistik und Geschäftsprozesse", HUSS, München, 2009

[Jod12] Jodin, Dirk: Grundlagen zum Effizienzvergleich in der Materialflusstechnik In: Tagungsband Logistikwerkstatt Graz 2012: Ressourceneffizienz in der technischen Logistik, Technischen Universität Graz, Graz, 21 und 22.06.2012

[Jun12] Jungheinrich AG: Das Jungheinrich Umweltprädikat, http://www.junghein rich.de/de/com/index-de/presse/umwelt -und-gesundheit/oekobilanz.html; Aufgerufen am 19.07.2012

[Kem12] Kemna, René; Azaïs, Nelly.; van Elburg, Martijn; van der Voort, Maaike; Li, William: MEErP Methodology Report Part 2 Final, http://www.meerp.eu/
[Kön11] König, Horst: Maschinen im Baubetrieb, Vieweg+Teubner, Wiesbaden, 2011

[LeD05] Le-Duc, Tho: Design and control of efficient order picking processes, Dissertation, Erasmus Research Institute of Management (ERIM) RSM Erasmus Iniveversity / Rotterdam School of Economics Erasmus University, Rotterdam, Rotterdam, 2005

[Mal91] Malton I.: Efficient order picking - The need for it and possivle solutions, Proceeding of the 11th International Conference Automation in Warehousing 11, 1991

[Mül98] Müller, Herbert W.: Die Umlaufgetriebe, Springer, Berlin, 1998

[Öko12] Öko-Insitut e.V., Umweltbundesamt: Prozessorientierte Basisdaten für Umweltmanagement-Instrumente

(PROBAS), http://www.probas.umwelt bundesamt.de/php/index.php? am 02.08.2012

[Sch12] Schischke, Karsten: Energy-using Product Group Analysis - Lot $5 \mathrm{Ma}$ chine Tools and related machinery Task 4 Report - Assessment of Base Case, Berlin, 2012

[Sch94] Scheffler, Martin: Grundlagen der Fördertechnik - Elemente und Triebwerke, Vieweg, Braunschweig / Wiesbaden, 1994

[Süd12] Süddeutsche Zeitung: Google verbraucht Strom für eine ganze Stadt, http://www.sueddeutsche.de/digital/ene rgiebilanz-des-internet-konzerns-google -verbraucht-strom-fuer-eine-ganze-stadt -1.1141053, 9.9.2011, am 02.05.2012

[VDI02] VDI-Richtlinie 2198: Typenblätter für Flurförderzeuge, VDI-Verlag, Düsseldorf, 2002

[Wis09] Wisser, Jens: Der Prozess Lagern und Kommissionieren im Rahmen des DCRM, Dissertation, Wissenschaftliche Berichte des Institutes für Fördertechnik und Logistiksysteme der Universität Karlsruhe (TH), Band 72, Karlsruhe, 2009 